\title{
The Irony of PET Tau Probe Specificity
}

Jorge R. Barrio

Department of Molecular and Medical Pharmacology, The David Geffen UCLA School of Medicine, Los Angeles, California

\section{See the associated article on page 117.}

$\mathbf{I}$ may be rather surprising that after more than 4 decades of PET development and thousands of molecular imaging probe candidates synthesized, relatively few among them are consistently used to investigate human diseases while even a much smaller number have reached the domain of patient care. There are very good reasons for this occurrence, however. One of the arguments being put forward focuses on the lack of appropriate synthetic methods for the development of PET biomarkers. However, the history of PET indicates that creative molecular probe design and rational translational evaluation has been the most significant limiting factor.

Tau-specific PET imaging probes are no exception to the above rationale. For instance, a ${ }^{18} \mathrm{~F}$-fluorinated analog belonging to a family of 5H-pyrido[4,3-b]indoles, known as T-807, has initially been touted as a tau-sensitive and presumably specific PET tau imaging probe. This PET probe candidate was selected from a chemical library of compounds screened primarily using in vitro binding to isolated fibrils and macro- and microautoradiography procedures (1). On the basis of these evaluations, T-807 was promptly accepted as a suitable candidate for in vivo use with PET, with little additional basic research investigations. The wide availability of this probe, courtesy of Eli Lilly \& Co., which trademarked it as AV-1451, may bear some responsibility for its hasty use to monitor tau deposition using PET in the living brains of Alzheimer disease $(\mathrm{AD})$ patients.

The connection between tau deposition and neuronal losses clearly provided a good opportunity for a logical approach to investigating disease progression and a possible diagnostic tool for $\mathrm{AD}$ using PET, conceptually superior to that resulting from PET monitoring of amyloid aggregates, the other pathognomonic neuroaggregate in $\mathrm{AD}$. Recognition of the significance by the National Institutes of Health soon resulted in generous funding for use of AV-1451 in living subjects with AD, but also in other predominant tauopathies, such as progressive supranuclear palsy and chronic traumatic encephalopathy, the latter having a 3-repeat (3R)/4-repeat (4R) tau isoform ratio similar to that of AD. The broad impact of chronic traumatic encephalopathy in society in

Received Aug. 23, 2017; revision accepted Aug. 23, 2017.

For correspondence or reprints contact: Jorge R. Barrio, UCLA School of Medicine, 10833 Le Conte Ave., Los Angeles, CA 90095-6948.

E-mail: jbarrio@mednet.ucla.edu

Published online Nov. 9, 2017.

COPYRIGHT @ 2018 by the Society of Nuclear Medicine and Molecular Imaging. DOI: 10.2967/jnumed.117.198960 general, and its close association to the ailments of many of the National Football League (American football) players, was also undoubtedly a driving force for this generous extramural support.

Within a short period, AV-1451 became the darling of tauspecific probes, but with diligent PET determinations in living patients, and additional postmortem determinations performed, unexpected results and inconsistencies clearly began to emerge. Notable among them were nonspecific, tau-unrelated binding in the choroid plexus and striatum, and low sensitivity for distinguishing AD cases from healthy controls (2). Also, whereas AV1451 was found to bind to the $3 R / 4 R$ tau isoforms present in $A D$, $3 \mathrm{R}$ and $4 \mathrm{R}$ tau isoforms typically forming straight filaments in predominant tauopathies such as progressive supranuclear palsy, MAPT P301 L mutation carriers, corticobasal degeneration, and frontotemporal dementia did not seem to be recognized by this PET biomarker (3). The same investigators had earlier reported (4) that autoradiography failed to show detectable AV-1451 binding in multiple brain regions examined postmortem in non-AD tauopathies, and further they also confirmed the presence of nonspecific binding of $\mathrm{AV}-1415$, among others, to neuromelanin and melanincontaining cells in the brain, as well as to brain hemorrhagic lesions.

Another comprehensive report (5) assessing AV-1451 binding to postmortem brain tissue from patients with a range of neurodegenerative diseases, tauopathies and nontauopathies, and normal controls concluded that "evidence for a non-tau binding site and lack of correlation between tracer binding and antibody staining suggested that in vivo quantification of tau load with T-807/AV1451 is problematic." This finding was preceded by the work of Vermeiren et al. (6) reporting that AV-1451 has a high nanomolar binding affinity for monoamine oxidase A (MAO-A) and MAO-B, enzymes abundantly present in the human brain in both neurons and glia, which contributed to the off-target in vivo AV-1451 PET signal. Displacement of [H-3]AV-1451 from its high-affinity-binding sites in human and rat brain homogenates with clorgyline, a selective MAO-A ligand, demonstrated unequivocally the off-target ligand binding to the enzyme.

Whereas MAO-A is widely distributed throughout the brain, MAO-B has been shown to be predominantly present in the basal ganglia and related subcortical structures. The consequences of this nonspecific, off-target binding of $\mathrm{AV}-1451$ are therefore significant. The cortical brain localization of MAO-A, in brain areas expected to have tau aggregates in various diseases, is a confounding factor and a severe limitation to any attempt to visualize and much less to quantify tau neuroaggregates with AV1451 in the same regions. On the other hand, it was the likely MAO-B-mediated nonspecific label in the basal ganglia with AV1451 that derailed the initial conclusions made on a single case of possible chronic traumatic encephalopathy in a football player, which was soon dismissed as erroneous (7). The work of Choi et al. (in this issue of The Journal of Nuclear Medicine (8)) provides 
additional important evidence that age-related off-target AV-1451 binding in the basal ganglia is also closely correlated with iron accumulation as measured using iron-sensitive $\mathrm{R}^{*} \mathrm{MR}$ imaging.

Therefore, most of the problems associated with AV-1451 in vivo use do not seem entirely related to the strenuous requirements of tau/amyloid aggregate binding specificity for molecular imaging probes (9), but most importantly due to its nonspecific binding to multiple tissue targets as indicated above. It may be surmised, however, that the possibility of T-807/AV-1451 binding to MAO enzymes could have been anticipated because it had already been known from the literature that $5 \mathrm{H}$-pyrido[4,3-b]indole was indeed a potent MAO inhibitor (10). However, this evidence was surprisingly unrecognized in the original publication (1), wherein it was reported that T-807/AV-1451 - a 5H-pyrido[4,3-b]indole-did not inhibit MAO-A or MAO-B.

It has also been a fundamental recognition that "claims of AV1451 high specificity for tau aggregates in AD were based primarily on autoradiography studies," which, as their design indicates, would fail to predict off-target labeling. Undoubtedly, in vitro experiments are an important first step in PET probe development, but the limitations of these methods have been clearly established earlier in the literature (11). Still, in vitro experiments, no matter how carefully designed, are never capable of fully simulating the in vivo environment, for which in vitro to in vivo extrapolations should always be made with extreme care.

Cautionary voices have recently been raised to express concern for the disappointing observations with AV-1451, as well as other tau imaging agents (e.g., THK-5351), in a call for a humbling approach, "to avoid making overarching claims that are supported by little data" (12). Also, as bluntly proclaimed, their lack of specificity may be "a kiss of death" for several molecular candidates (12) and it would be hard to disagree with the sagely expressed opinions of multiple investigators on the gloomy outcome of the so-called first generation of tau-specific imaging agents. These results with AV-1451 (and other purported tau-specific imaging probes) should give us pause to fully comprehend the difficulties of PET probe development (11). Just injecting a radioactive compound with a limited and cursory validation to get a PET image is unfortunate with insightful PET biomarker development in order to get meaningful information.

\section{REFERENCES}

1. Xia CF, Arteaga J, Chen G, et al. $\left[{ }^{18} \mathrm{~F}\right] \mathrm{T} 807$, a novel tau positron emission tomography imaging agent for Alzheimer's disease. Alzheimers Dement. 2013; 9:666-676.

2. At HAI, researchers explore diagnostic potential of a tau tracer. Alzforum website. http://www.alzforum.org/news/conference-coverage/hai-researchers-explorediagnostic-potential-tau-tracer. February 10, 2016. Accessed November 30, 2017.

3. Marquié M, Normandin MD, Meltzer AC, et al. Pathological correlations of [F-18]AV-1451 imaging in non-Alzheimer tauopathies. Ann Neurol. 2017;81:117-128.

4. Marquié M, Normandin MD, Vanderburg CR, et al. Validating novel tau positron emission tomography tracer [F-18]-AV-1451 (T807) on postmortem brain tissue. Ann Neurol. 2015;78:787-800.

5. Sander K, Lashley T, Gami P, et al. Characterization of tau positron emission tomography tracer $\left[{ }^{18} \mathrm{~F}\right] \mathrm{AV}-1451$ binding to postmortem tissue in Alzheimer's disease, primary tauopathies, and other dementias. Alzheimers Dement. 2016;12: 1116-1124.

6. Vermeiren C, Mercier J, Viot D, et al. The tau tracer T807 binds with nanomolar affinity to monoamine oxidase enzyme. Alzheimers Dement. 2015;11(suppl):283.

7. Gandy S, DeKosky ST. $\left[{ }^{18} \mathrm{~F}\right]-\mathrm{T} 807$ tauopathy PET imaging in chronic traumatic encephalopathy. F1000Res. 2014;3:229.

8. Choi JY, Cho H, Ahn SJ, et al. Off-target ${ }^{18} \mathrm{~F}-\mathrm{AV}-1451$ binding in the basal ganglia correlates with age-related iron accumulation. J Nucl Med. 2018;59:117-120.

9. Schafer KN, Kim SA, Matzavinos A, Kuret J. Selectivity requirements for diagnostic imaging of neurofibrillary lesions in Alzheimer's disease: a simulation study. Neuroimage. 2012;60:1724-1733.

10. Maruyama W, Ota A, Takahashi A, Nagatsu T, Naoi M. Food-derived heterocyclic amines, 3-amino-1,4-dimethyl-5H-pyrido[4,3-b]indole and related amines, as inhibitors of monoamine metabolism. J Neural Transm Suppl. 1994;41:327-333.

11. Frey KA, Albin RL. Receptor binding techniques. Curr Protoc Neurosci. 2001; chapter1:unit 1.4.

12. Next-generation tau PET tracers strut their stuff. Alzforum website. http:// www.alzforum.org/news/conference-coverage/next-generation-tau-pet-tracersstrut-their-stuff\#comment-23856. April 14, 2017. Accessed November 30, 2017. 